\title{
Continuous-time random walk for open systems: Fluctuation theorems and counting statistics
}

\author{
Massimiliano Esposito* and Katja Lindenberg \\ Department of Chemistry and Biochemistry and Institute for Nonlinear Science, University of California, San Diego, \\ La Jolla, California 92093-0340, USA
}

(Received 5 February 2008; published 19 May 2008)

\begin{abstract}
We consider continuous-time random walks (CTRW) for open systems that exchange energy and matter with multiple reservoirs. Each waiting time distribution (WTD) for times between steps is characterized by a positive parameter $\alpha$, which is set to $\alpha=1$ if it decays at least as fast as $t^{-2}$ at long times and therefore has a finite first moment. A WTD with $\alpha<1$ decays as $t^{-\alpha-1}$. A fluctuation theorem for the trajectory quantity $R$, defined as the logarithm of the ratio of the probability of a trajectory and the probability of the time reversed trajectory, holds for any CTRW. However, $R$ can be identified as a trajectory entropy change only if the WTDs have $\alpha=1$ and satisfy separability (also called "direction time independence"). For nonseparable WTDs with $\alpha=1, R$ can only be identified as a trajectory entropy change at long times, and a fluctuation theorem for the entropy change then only holds at long times. For WTDs with $0<\alpha<1$ no meaningful fluctuation theorem can be derived. We also show that the (experimentally accessible) $n$th moments of the energy and matter transfers between the system and a given reservoir grow as $t^{n \alpha}$ at long times.
\end{abstract}

DOI: 10.1103/PhysRevE.77.051119

PACS number(s): 05.70.Ln, 05.40.Fb, 05.40.-a

\section{INTRODUCTION}

It has long been clearly understood that the statement of the second law of thermodynamics concerning the increase in entropy in an isolated system as it goes to equilibrium refers only to the average behavior, but this was sufficient as long as one dealt only with macroscopic systems characterized by extremely narrow ensemble distributions with fluctuations that were essentially never observed. More recently, with the ability to quantitatively monitor systems on the extremely small scales of single molecules and quantum dots, it is possible to study fluctuations around the average behavior. Fluctuation theorems that hold arbitrarily far from equilibrium have thus become subject to experimental verification [1-7]. These theorems in general deal with the ratio of the probabilities of a given system trajectory and that of its time reversed trajectory, either as the system goes to equilibrium or as it evolves to a steady state under the action of nonequilibrium constraints imposed on the system. From this one can calculate, for example, the relative probabilities that the entropy of an isolated system away from thermodynamic equilibrium will spontaneously increase or decrease over a given period of time. The ratio is essentially infinite in a macroscopic system away from equilibrium and is unity due to fluctuations in equilibrium, but in sufficiently small systems away from equilibrium it is merely large (and experimentally accessible) rather than infinite.

Fluctuation theorems can take different forms depending on the specific problem under consideration, but they are all ultimately connected to the probabilistic asymmetry of system trajectories and time reversed trajectories. Equilibrium corresponds to the situation where the symmetry is restored as stated by the principle of microreversibility. Fluctuation

\footnotetext{
*Also at Center for Nonlinear Phenomena and Complex Systems, Universite Libre de Bruxelles, Code Postal 231, Campus Plaine, B-1050 Brussels, Belgium.
}

theorems have been formulated for a wide range of dynamics such as driven Hamiltonian dynamics [8-10], stochastic dynamics [11-16], deterministic thermostated dynamics $[17,18]$, and even quantum dynamics [19-24]. Here we focus on stochastic dynamics in an effort to explore the validity of fluctuation theorems beyond the stochastic dynamics that have been considered to date.

In this narrower context of stochastic dynamics, most previous studies of fluctuation theorems have focused on systems described by Markovian master equations or FokkerPlanck equations. Recently there have been some efforts to investigate fluctuation theorems for systems described by nonlinear generalized Langevin equations [25,26] with an external driving force as a nonequilibrium constraint. Our focus is on nonequilibrium systems described by continuoustime random walks (CTRW) [27-32] in which transitions between microscopic states may be caused by more than one mechanism. The nonequilibirum constraint is imposed when these mechanisms have different statistical properties such as, for example, through contact with two heat baths maintained at different temperatures. In general, identifying such nonequilibrium constraints may itself be complicated $[15,33]$, and we will here explicitly point to these differences.

We pose the following question: What properties of a CTRW are necessary for an entropy fluctuation theorem to be valid under such nonequilibrium constraints? We note, for example, that CTRWs are known to display aging [34-36] as well as nonergodic phenomena $[37,38]$ which may significantly alter the behavior of the system under time reversal and prevent a fluctuation theorem from being satisfied. At the same time, CTRWs under certain conditions reduce to Markovian master equations which are known to satisfy fluctuation theorems. CTRWs therefore provide a good framework to study the validity of fluctuation theorems. In particular, our results will hopefully contribute clarification to recent observations of anomalous statistics in the nonequilibrium fluctuations of single molecules and quantum dots [39-42]. 
A second purpose of this paper is the formulation of a general framework for the calculation of (experimentally accessible) counting statistics of events associated with a given mechanism. Examples of such events might involve particle or energy transfer. To accomplish this we use a method based on the propagation of the generating function associated with the probability distribution of the events, in the spirit of the method used for Markovian master equations $[12,16]$. This will allow us to investigate the long-time behavior of the moments of the distribution associated with the counting statistics.

Our basic CTRW model is constructed as follows. We consider a stochastic dynamics between microscopic states $m$ of a system with a finite number of states. The transitions between states may be due to different mechanisms $\nu$. For example, we will subsequently consider a system in which each microscopic state $m$ is characterized by a number $N_{m}$ of particles and an energy $\epsilon_{m}$, and where the transitions between the $m$ 's are triggered by different reservoirs (heat baths) $\nu$. Suppose that the system arrives at state $m^{\prime}$ at a given time and that its next jump is to state $m$ at a time $t$ later via mechanism $\nu$. The distribution of waiting times (WTD) for this to occur is denoted by $\psi_{m m^{\prime}}^{(\nu)}(t)$, with other related quantities specified in more detail subsequently. We focus on waiting time distributions whose long-time behavior is reflected in the small-s Laplace transform

$$
\tilde{\psi}_{m m^{\prime}}^{(\nu)}(s) \underset{s \rightarrow 0}{=} P_{m m^{\prime}}^{(\nu)}-B_{m m^{\prime}}^{(\nu)} s^{\alpha},
$$

where $\widetilde{f}(s) \equiv \int_{0}^{\infty} d t \mathrm{e}^{-s t} f(t)$ and $0<\alpha \leq 1$. The $B_{m m^{\prime}}^{(\nu)}$ are elements of an arbitrary matrix. A detailed discussion surrounding this choice can be found in [43]. When $0<\alpha<1$ the long-time decay of the WTDs is then of the power-law form $\psi_{m m^{\prime}}^{(\nu)}(t) \sim t^{-\alpha-1}$. When $\alpha=1$ the decay is at least as fast as $1 / t^{2}$ but may be faster.

In Sec. II we present the CTRW model for an open system driven by different mechanisms described by different statistical properties, and formally express the probability that the system is in state $m$ at time $t$. In Sec. III we derive the generalized master equation satisfied by this probability and study its long-time behavior. In Sec. IV we present a generating function formalism to calculate the probability distribution of heat and matter transfers which is used to study the long-time behavior of the moments of the distribution. The principal results of this work, namely, the conditions for the validity of fluctuation theorems, are presented in Sec. V. In particular, we show that fluctuation theorems for the entropy change can only be obtained if $\alpha=1$, that is, if the WTDs decay at least as fast as $t^{-2}$ at long times. Furthermore, even in this case the entropy change can be expressed as a familiar ratio of the probability of a trajectory and its time reversed trajectory only if the WTDs satisfy constraints of separability. A summary of results and some concluding remarks are presented in Sec. VI.

\section{CONTINUOUS-TIME RANDOM WALKS FOR OPEN SYSTEMS}

Our goal in this section is to construct the probability that the system will be found in a particular state at time $t$. Sup- pose that the system arrives at state $m^{\prime}$ at time zero, and that its next jump is to state $m$ at time $t$ via mechanism $\nu$. The waiting time distribution $\psi_{m m^{\prime}}^{(\nu)}(t)$ for this event introduced earlier satisfies the normalization condition

$$
\int_{0}^{\infty} d \tau \sum_{m, \nu} \psi_{m m^{\prime}}^{(\nu)}(\tau)=1
$$

For convenience, we define $\psi_{m m}^{(\nu)}(\tau) \equiv 0$. The probability that no transition occurs up to time $t$ after arrival at $m^{\prime}$ at time zero is

$$
\phi_{m^{\prime}}(t)=\sum_{m, \nu} \int_{t}^{\infty} d \tau \psi_{m m^{\prime}}^{(\nu)}(\tau)=1-\sum_{m, \nu} \int_{0}^{t} d \tau \psi_{m m^{\prime}}^{(\nu)}(\tau) .
$$

We see that by construction $\phi_{m^{\prime}}(\infty)=0$, that is, a jump eventually occurs with certainty. We define the auxiliary distributions

$$
\begin{aligned}
\psi_{m^{\prime}}^{(\nu)}(t) & \equiv \sum_{m} \psi_{m m^{\prime}}^{(\nu)}(t), \\
\psi_{m^{\prime}}(t) & \equiv \sum_{\nu} \psi_{m^{\prime}}^{(\nu)}(t) .
\end{aligned}
$$

$\psi_{m^{\prime}}^{(\nu)}(t)$ is the waiting time distribution of the first jump from state $m^{\prime}$ to any other state via a mechanism $\nu$, and $\psi_{m^{\prime}}(t)$ is the waiting time distribution of the first jump from state $m^{\prime}$ to any other state regardless of the mechanism, given that arrival at $m^{\prime}$ occurred at time zero. We also define

$$
\begin{gathered}
P_{m m^{\prime}}^{(\nu)} \equiv \int_{0}^{\infty} d \tau \psi_{m m^{\prime}}^{(\nu)}(\tau), \\
P_{m m^{\prime}} \equiv \sum_{\nu} P_{m m^{\prime}}^{(\nu)} .
\end{gathered}
$$

Here $P_{m m^{\prime}}^{(v)}$ is the probability that, being at $m^{\prime}$, the next jump will be from state $m^{\prime}$ to $m$ via mechanism $\nu$, and $P_{m m^{\prime}}$ is the probability that the jump will be from $m^{\prime}$ to $m$ irrespective of the mechanism. Note that by definition $P_{m m} \equiv 0$. We finally define the probability $f_{m^{\prime}}^{(\nu)}$ that the next jump from $m^{\prime}$ will be due to mechanism $\nu$,

$$
f_{m^{\prime}}^{(\nu)} \equiv \sum_{m} P_{m m^{\prime}}^{(\nu)}
$$

The normalization condition (2) implies

$$
\begin{gathered}
\int_{0}^{\infty} d \tau \psi_{m}(t)=1 \\
\sum_{m} P_{m m^{\prime}}=1 \\
\sum_{\nu} f_{m^{\prime}}^{(v)}=1 .
\end{gathered}
$$

The determining feature of the problem for our purposes is the behavior of the first moment of the waiting time distribution $\psi_{m m^{\prime}}^{(\nu)}(t)$ and, in particular, whether it is finite or infi- 
nite. This first moment is just the average time that the system remains in state $m^{\prime}$ before jumping to $m$ via mechanism $\nu$ :

$$
t_{m m^{\prime}}^{(\nu)} \equiv \int_{0}^{\infty} d \tau \tau \psi_{m m^{\prime}}^{(\nu)}(\tau)
$$

Associated mean waiting times are given by

$$
\begin{aligned}
t_{m^{\prime}}^{(\nu)} & \equiv \sum_{m} t_{m m^{\prime}}^{(\nu)}, \\
t_{m^{\prime}} & \equiv \sum_{\nu} t_{m^{\prime}}^{(\nu)} .
\end{aligned}
$$

The first is the average time that the system remains in state $m^{\prime}$ without jumping anywhere else via mechanism $\nu$. The second is the average time that the system remains at $m^{\prime}$ without making any jumps at all by any mechanism. If $\alpha$ $<1$, all moments of the waiting time distribution (including the first moments or mean waiting times) are divergent, whereas for $\alpha=1$ the first moments $t_{m m^{\prime}}^{(\nu)}$ are finite. In this case, from Eqs. (1) and (8) it follows that $B_{m m^{\prime}}^{(\nu)}=t_{m m^{\prime}}^{(\nu)}$.

Suppose that we begin our observations at time $t=0$, at which time we find the system in state $m^{\prime}$. Since in general we may not know when the jump occurred that brought the system to that state, we need to distinguish the waiting time distribution of the first jump after time zero from that of subsequent jumps. We mark this first waiting time distribution with a prime, $\psi_{m m^{\prime}}^{\prime(v)}(t)$. The probability that no transition away from $m^{\prime}$ occurs up to time $t$ must be similarly distinguished, $\phi_{m}^{\prime}(t)$. The primed functions are equal to the unprimed ones only if a jump occurred exactly at time zero or if the WTDs decay exponentially. If there is no information about when the last jump before time $t=0$ occurred, then the primed functions can be related to the unprimed ones only if the mean waiting times $t_{m m^{\prime}}^{(\nu)}$ are finite. In this case, an average over the uncertain past yields

$$
\begin{aligned}
\psi_{m m^{\prime}}^{\prime(\nu)}(t) & =\frac{\int_{-\infty}^{0} d \tau \psi_{m m^{\prime}}^{(\nu)}(t-\tau)}{\sum_{m, \nu} \int_{0}^{\infty} d t \int_{-\infty}^{0} d \tau \psi_{m m^{\prime}}^{(\nu)}(t-\tau)} \\
& =\frac{\int_{-\infty}^{0} d \tau \psi_{m m^{\prime}}^{(\nu)}(t-\tau)}{t_{m^{\prime}}}=\frac{\int_{t}^{\infty} d \tau \psi_{m m^{\prime}}^{(\nu)}(\tau)}{t_{m^{\prime}}} .
\end{aligned}
$$

Note that summing Eq. (10) over $m$ and $\nu$ and using Eq. (3) leads to

$$
\psi_{m^{\prime}}^{\prime}(t)=\frac{\phi_{m^{\prime}}(t)}{t_{m^{\prime}}}
$$

Similarly,

$$
\phi_{m^{\prime}}^{\prime}(t)=\frac{\int_{t}^{\infty} d \tau \phi_{m^{\prime}}(\tau)}{t_{m^{\prime}}} .
$$

It is straightforward to construct an integral equation for $\rho_{m}(t)$, the probability that the system is in state $m$ at time $t$. For this purpose we also define $\eta_{m}(t)$, the probability that the system jumps onto state $m$ at time $t$. The following integral CTRW relations are evident [27-30,32]:

$$
\eta_{m}(t)=\sum_{m^{\prime}, \nu} \psi_{m m^{\prime}}^{(\nu)}(t) \rho_{m^{\prime}}(0)+\sum_{m^{\prime}, \nu} \int_{0}^{t} d \tau \psi_{m m^{\prime}}^{(\nu)}(t-\tau) \eta_{m^{\prime}}(\tau)
$$

and

$$
\rho_{m}(t)=\phi_{m}^{\prime}(t) \rho_{m}(0)+\int_{0}^{t} d \tau \phi_{m}(t-\tau) \eta_{m}(\tau) .
$$

Since these are convolutions, it is easiest to solve for the Laplace transform $\tilde{\rho}_{m}(s)$ of the desired probability $\rho_{m}(t)$. The Laplace transforms of these two relations are

$$
\widetilde{\eta}_{m}(s)=\sum_{m^{\prime}, \nu}\left[\widetilde{\psi}_{m m^{\prime}}^{\prime(\nu)}(s) \rho_{m^{\prime}}(0)+\widetilde{\psi}_{m, m^{\prime}}^{(\nu)}(s) \widetilde{\eta}_{m^{\prime}}(s)\right],
$$

and

$$
\tilde{\rho}_{m}(s)=\widetilde{\phi}_{m}^{\prime}(s) \rho_{m}(0)+\widetilde{\phi}_{m}(s) \tilde{\eta}_{m}(s) .
$$

The solution is most neatly expressed in terms of the matrices $\Phi$ and $\Psi$ with matrix elements

$$
\begin{gathered}
{[\widetilde{\Psi}(s)]_{m m^{\prime}} \equiv \widetilde{\psi}_{m, m^{\prime}}^{\prime}(s),} \\
{[\widetilde{\Phi}(s)]_{m m^{\prime}} \equiv \delta_{m m^{\prime}} \widetilde{\phi}_{m}(s),}
\end{gathered}
$$

and the vectors $|\widetilde{\eta}(s)\rangle$ and $|\widetilde{\rho}(s)\rangle$ with elements $\widetilde{\eta}_{m}(s)$ and $\tilde{\rho}_{m}(s)$. The solution of Eq. (15) is

$$
|\widetilde{\eta}(s)\rangle=[I-\widetilde{\Psi}(s)]^{-1} \widetilde{\Psi}^{\prime}(s)|\rho(0)\rangle,
$$

where $I$ is the identity matrix. Using Eq. (16), the formal solution of the CTRW then is

$$
|\widetilde{\rho}(s)\rangle=\left\{\tilde{\Phi}^{\prime}(s)+\widetilde{\Phi}(s)[I-\tilde{\Psi}(s)]^{-1} \tilde{\Psi}^{\prime}(s)\right\}|\rho(0)\rangle .
$$

\section{GENERALIZED MASTER EQUATION}

In this section we construct the generalized master equation for the probability that the system is in state $m$ at time $t$. This equation facilitates the exploration of the behavior of this probability at long times. Using the Laplace transform of Eq. (3),

$$
\widetilde{\phi}_{m}(s)=\frac{1}{s} \sum_{m^{\prime}}\left[\delta_{m m^{\prime}}-\widetilde{\psi}_{m^{\prime} m}(s)\right]=\frac{1}{s}\left[1-\tilde{\psi}_{m}(s)\right],
$$

we can rewrite Eq. (16) as 


$$
\widetilde{\rho}_{m}(s)=\widetilde{\phi}^{\prime}(s) \rho_{m}(0)+\frac{1}{s} \widetilde{\eta}_{m}(s)-\frac{1}{s} \sum_{m^{\prime}, \nu} \widetilde{\psi}_{m^{\prime} m}^{(\nu)}(s) \widetilde{\eta}_{m}(s) .
$$

Inserting Eq. (15) in the second term of Eq. (21) and using Eq. (16), we obtain

$$
s \widetilde{\rho}_{m}(s)-\rho_{m}(0)=\widetilde{\mathcal{I}}(s)+\sum_{m^{\prime}, \nu}\left[\widetilde{W}_{m m^{\prime}}^{(\nu)}(s) \widetilde{\rho}_{m^{\prime}}(s)-\widetilde{W}_{m^{\prime} m}^{(\nu)}(s) \widetilde{\rho}_{m}(s)\right],
$$

where the transition matrix elements are given by

$$
\widetilde{W}_{m m^{\prime}}^{(\nu)}(s)=\frac{\widetilde{\psi}_{m m^{\prime}}^{(\nu)}(s)}{\widetilde{\phi}_{m^{\prime}}(s)}
$$

and

$$
\begin{aligned}
\tilde{\mathcal{I}}(s) \equiv & \sum_{m^{\prime}, \nu}\left\{\tilde{W}_{m m^{\prime}}^{(\nu)}(s)\left[\widetilde{\phi}_{m^{\prime}}(s)-\widetilde{\phi}_{m^{\prime}}^{\prime}(s)\right] \rho_{m^{\prime}}(0)\right. \\
& \left.-\widetilde{W}_{m^{\prime} m}^{(\nu)}(s)\left[\widetilde{\phi}_{m}(s)-\widetilde{\phi}_{m}^{\prime}(s)\right] \rho_{m}(0)\right\} .
\end{aligned}
$$

The inhomogeneous term $\tilde{\mathcal{I}}(s)$ in Eq. (22) thus depends on the initial condition. It vanishes if a jump occurs at time zero or if the waiting time distributions are exponential because then $\phi_{m}^{\prime}(t)=\phi_{m}(t)$. Upon inverse Laplace transformation we arrive at the generalized master equation

$$
\begin{aligned}
\dot{\rho}_{m}(t)= & \mathcal{I}(t)+\sum_{m^{\prime}, \nu} \int_{0}^{t} d \tau\left[W_{m m^{\prime}}^{(\nu)}(\tau) \rho_{m^{\prime}}(t-\tau)\right. \\
& \left.-W_{m^{\prime} m}^{(\nu)}(\tau) \rho_{m}(t-\tau)\right] .
\end{aligned}
$$

The generalized master equation is clearly non-Markovian unless $\tilde{W}_{m m^{\prime}}^{(\nu)}(s)$ is independent of $s$. This occurs, for example, for separable distributions $\psi_{m m^{\prime}}^{(\nu)}(t)=P_{m m^{\prime}}^{(\nu)} \psi_{m^{\prime}}(t)$ with $\psi_{m}(t)$ $=e^{-t / t_{m}} / t_{m}$. Indeed, in this case $\widetilde{W}_{m m^{\prime}}^{(\nu)}(s)=P_{m m^{\prime}}^{(\nu)} / t_{m^{\prime}}$.

Of interest for our purposes is the long-time behavior of the probability $\rho_{m}(t)$. Here we distinguish the case $\alpha=1$, associated with finite mean waiting times, from the case 0 $<\alpha<1$, associated with divergent mean waiting times.

\section{A. Long-time behavior for $\alpha=1$}

Consider first the long-time behavior of the generalized master equation in the case $\alpha=1$. From Eq. (20) it follows that

$$
\widetilde{\phi}_{m}(s) \underset{s \rightarrow 0}{=B_{m} s^{\alpha-1},}
$$

where $B_{m}=\sum_{m^{\prime}, \nu} B_{m^{\prime} m}^{(\nu)}$. Since it follows from this result and from Eq. (1) that $B_{m m^{\prime}}=t_{m m^{\prime}}$ and $B_{m}=t_{m}$, Eq. (23) then immediately leads to

$$
\widetilde{W}_{m m^{\prime}}^{(\nu)}(0)=\lim _{s \rightarrow 0} \widetilde{W}_{m m^{\prime}}^{(\nu)}(s)=\frac{\widetilde{\psi}_{m m^{\prime}}^{(\nu)}(0)}{\widetilde{\phi}_{m^{\prime}}(0)}=\frac{P_{m m^{\prime}}^{(\nu)}}{t_{m^{\prime}}} .
$$

Since $\lim _{s \rightarrow 0}\left(\widetilde{\phi}_{m^{\prime}}(s)-\widetilde{\phi}_{m^{\prime}}^{\prime}(s)\right)$ is constant when $\alpha=1$, we find that $\lim _{s \rightarrow 0} \mathcal{I}(s)$ is also constant [see Eq. (24)]. Therefore, using the final value theorem $f(\infty)=\lim _{s \rightarrow 0} s \tilde{f}(s)[32,45]$, we find that

$$
\lim _{t \rightarrow \infty} \mathcal{I}(t)=0,
$$

$$
\lim _{t \rightarrow \infty} W_{m m^{\prime}}^{(\nu)}(t)=0
$$

This means that at long times, the generalized master equation behaves like the Markovian master equation

$$
\dot{\rho}_{m}(t)=\sum_{m^{\prime}, \nu}\left[\widetilde{W}_{m m^{\prime}}^{(\nu)}(0) \rho_{m^{\prime}}(t)-\widetilde{W}_{m^{\prime} m}^{(\nu)}(0) \rho_{m}(t)\right] .
$$

Defining the rate matrix $\boldsymbol{V} \equiv \Sigma_{\nu} \boldsymbol{V}^{(\nu)}$ where

$$
\begin{gathered}
V_{m m^{\prime}}^{(\nu)} \equiv \frac{P_{m m^{\prime}}^{(\nu)}}{B_{m^{\prime}}} \quad \text { for } m \neq m^{\prime}, \\
V_{m m}^{(\nu)} \equiv-\sum_{m^{\prime}(\neq m)} V_{m^{\prime} m}^{(\nu)},
\end{gathered}
$$

we can rewrite Eq. (29) as $|\dot{\rho}(t)\rangle=\boldsymbol{V}|\rho(t)\rangle$. Using the PerronFrobenious theorem, all eigenvalues of $\boldsymbol{V}$ are negative aside from one which is zero. The probability $\rho_{m}(t)$ therefore decays exponentially to a steady state solution $\rho_{m}^{\mathrm{ss}}$ that obeys the condition

$$
\sum_{m^{\prime}, \nu}\left[\tilde{W}_{m m^{\prime}}^{(\nu)}(0) \rho_{m^{\prime}}^{\mathrm{ss}}-\tilde{W}_{m^{\prime} m}^{(\nu)}(0) \rho_{m}^{\mathrm{ss}}\right]=0
$$

The steady state solution corresponds to equilibrium if the detailed balance condition

$$
\widetilde{W}_{m m^{\prime}}^{(\nu)}(0) \rho_{m^{\prime}}^{\mathrm{eq}}=\widetilde{W}_{m^{\prime} m}^{(\nu)}(0) \rho_{m}^{\mathrm{eq}}
$$

is satisfied, that is, if all fluxes between pairs of states associated with the different mechanisms $\nu$ become zero at equilibrium. This would not be possible if the long-time statistics of the different mechanisms were different. If the detailed balance condition is not satisfied, then the solution $\rho_{m m}^{\mathrm{ss}}$ is a nonequilibrium steady state.

A useful connection to thermodynamics is provided if we consider that each state $m$ has a given energy $\epsilon_{m}$ and number of particles $N_{m}$ and that each different mechanism $\nu$ inducing transitions between states corresponds to a given reservoir with a given temperature $\beta_{\nu}^{-1}$ and chemical potential $\mu_{\nu}$. We then assume that 


$$
\frac{\tilde{W}_{m m^{\prime}}^{(\nu)}(0)}{\tilde{W}_{m^{\prime} m}^{(\nu)}(0)}=\exp \left\{-\beta_{\nu}\left(\epsilon_{m}-\epsilon_{m^{\prime}}\right)+\beta_{\nu} \mu_{\nu}\left(N_{m}-N_{m^{\prime}}\right)\right\} .
$$

Equation (32) holds only if all the $\beta_{\nu}$ 's and $\mu_{\nu}$ 's are equal. In this case the equilibrium distribution corresponds to the grand canonical ensemble. If the $\beta_{\nu}$ 's and $\mu_{\nu}$ 's are different, the steady state is a nonequilibrium steady state that obeys Eq. (31).

\section{B. Long-time behavior for $0<\alpha<1$}

When $0<\alpha<1$, we must separately specify the statistical properties of the waiting time distribution for the first jump after $t=0$. We choose $\widetilde{\phi}_{m}^{\prime}(s)=\widetilde{\phi}_{m}(s)$ because other choices add only further complications but little of general interest to our specific problem. The inhomogeneous initial condition term then drops out, and for small $s$, using Eq. (30), Eq. (22) becomes

$$
s \widetilde{\rho}_{m}(s)-\rho_{m}(0)=\sum_{m^{\prime}, \nu} V_{m m^{\prime}}^{(\nu)} s^{1-\alpha} \widetilde{\rho}_{m^{\prime}}(s) .
$$

Using the rules of fractional calculus [44], this equation can be written in the time domain as

$$
\frac{d}{d t} \rho_{m}(t)=\sum_{m^{\prime}, \nu} V_{m m^{\prime} 0}^{(\nu)} D_{t}^{1-\alpha} \rho_{m^{\prime}}(t),
$$

where the Riemann-Liouville fractional integral is given by

$$
{ }_{0} D_{t}^{1-\alpha} \rho_{m^{\prime}}(t)=\frac{1}{\Gamma(\alpha)} \frac{d}{d t} \int_{0}^{t} d \tau(t-\tau)^{\alpha-1} \rho_{m^{\prime}}(\tau) .
$$

If the matrices with elements $V_{m m^{\prime}}^{(v)}$, can be diagonalized and the eigenvalues $v_{i}$ (which are all negative or zero) are nondegenerate, the solution of Eq. (35) can be written as a linear combination of solutions of

$$
\frac{d}{d t} \rho_{i}(t)=v_{i 0} D_{t}^{1-\alpha} \rho_{i}(t) .
$$

The solution of this equation is the Mittag-Leffler function $E_{\alpha}\left[-(t / \tau)^{\alpha}\right]$, where $\tau=\left(-v_{i}\right)^{1 / \alpha}$ (see Appendix B of Ref. [44]). At long times the Mittag-Leffler function decays as a power law,

$$
E_{\alpha}\left[-(t / \tau)^{\alpha}\right] \sim\left[(t / \tau)^{\alpha} \Gamma(1-\alpha)\right]^{-1}
$$

(at short times it behaves as a stretched exponential, $\left.E_{\alpha}\left[-(t / \tau)^{\alpha}\right] \sim \exp \left(-\frac{(t / \tau)^{\alpha}}{\Gamma(1+\alpha)}\right)\right)$. Thus the general solution of the generalized master equation is a linear combination of Mittag-Leffler functions, and the probability $\rho_{m}(t)$ decays toward the zero eigenvalue mode as a power law.

\section{COUNTING STATISTICS}

Although our ultimate goal is to establish conditions under which fluctuation theorems are valid for systems whose dynamics are described by CTRWs, we first consider the counting statistics for such a system. These statistics are interesting because they are experimentally accessible, and this analysis leads to some definitions that are useful in the discussion of fluctuation theorems.

Consider a system described by the CTRW of Sec. II where each microscopic state $m$ has a given number of particles $N_{m}$ and an energy $\boldsymbol{\epsilon}_{m}$, and where the allowed transitions between pairs of states are due to different mechanisms $\nu$, each corresponding to a reservoir $\nu$. We want to calculate the probability $P\left(\left\{\Delta E^{(\nu)}\right\},\left\{\Delta N^{(\nu)}\right\}, t\right)$ that an energy transfer $\Delta E^{(\nu)}$ and a matter transfer $\Delta N^{(\nu)}$ occurs between the system and the reservoir $\nu$ during time $t$. We define the set of parameters $\gamma \equiv\left(\left\{\gamma_{\mathrm{e}}^{(\nu)}\right\},\left\{\gamma_{\mathrm{m}}^{(\nu)}\right\}\right)$ and the generating function

$$
\begin{aligned}
G(i \gamma, t)= & \int_{-\infty}^{\infty}\left\{d \Delta E^{(\nu)}\right\} \sum_{\left\{\Delta N^{(\nu)}\right\}=-\infty}^{\infty} P\left(\left\{\Delta E^{(\nu)}\right\},\left\{\Delta N^{(\nu)}\right\}, t\right) \\
& \times \exp \left[i\left(\gamma_{\mathrm{e}}^{(\nu)} \Delta E^{(\nu)}+\gamma_{\mathrm{m}}^{(\nu)} \Delta N^{(\nu)}\right)\right] .
\end{aligned}
$$

The probability can be recovered from the generating function using

$$
\begin{aligned}
P\left(\left\{\Delta E^{(\nu)}\right\},\left\{\Delta N^{(\nu)}\right\}, t\right)= & \int_{-\infty}^{\infty}\left\{\frac{d \gamma_{\mathrm{e}}^{(\nu)}}{2 \pi}\right\} \int_{0}^{2 \pi}\left\{\frac{d \gamma_{\mathrm{m}}^{(\nu)}}{2 \pi}\right\} \\
& \times \exp \left\{-i\left(\Delta E^{(\nu)} \gamma_{\mathrm{e}}^{(\nu)}+\Delta N^{(\nu)} \gamma_{\mathrm{m}}^{(\nu)}\right)\right\} \\
& \times G(i \gamma, t) .
\end{aligned}
$$

Derivatives of the generating function with respect to the elements of $\boldsymbol{\gamma}$ evaluated at $\boldsymbol{\gamma}=0$ gives the moments of the distribution of this process.

We define

$$
\psi_{m m^{\prime}}(\gamma, t) \equiv \sum_{\nu} e^{\gamma_{\mathrm{m}}^{(\nu)}\left(N_{m}-N_{m^{\prime}}\right)} e^{\gamma_{\mathrm{e}}^{(\nu)}\left(\epsilon_{m^{-}}-\epsilon_{m^{\prime}}\right)} \psi_{m m^{\prime}}^{(\nu)}(t),
$$

which is the WTD matrix whose elements associated with a transition caused by mechanism $\nu$ are weighted by the exponential of $\gamma_{\mathrm{e}}^{(\nu)}\left(\gamma_{\mathrm{m}}^{(\nu)}\right)$ times the change of energy (matter) that this transition induces. We note that $\Psi(\gamma=0, t)=\Psi(t)$. To evaluate the generating function and the associated moments, we replace the WTD $\psi_{m m^{\prime}}^{(\nu)}(t)$ by Eq. (41) in the CTRW (13) and (14), and thus obtain

$$
\begin{aligned}
\eta_{m}(\boldsymbol{\gamma}, t)= & \sum_{m^{\prime} \nu} \psi_{m m^{\prime}}^{\prime(\nu)}(\boldsymbol{\gamma}, t) \rho_{m^{\prime}}(0) \\
& +\sum_{m^{\prime} \nu} \int_{0}^{t} d \tau \psi_{m m^{\prime}}^{(\nu)}(\boldsymbol{\gamma}, t-\tau) \eta_{m^{\prime}}(\boldsymbol{\gamma}, \tau)
\end{aligned}
$$

and

$$
\rho_{m}(\gamma, t)=\phi_{m}^{\prime}(t) \rho_{m}(0)+\int_{0}^{t} d \tau \phi_{m}(t-\tau) \eta_{m}(\gamma, \tau) .
$$

By doing so, we are weighting the probability of all the trajectories of length $t$ ending up in the state $m$ and along which a transfer of energy (matter) between the system and the reservoir $\nu$ occurs, by the exponential of $\gamma_{\mathrm{e}}^{(\nu)}\left(\gamma_{\mathrm{m}}^{(\nu)}\right)$ times this energy (matter) transfer. By summing over all final states $m$, we reconstruct the generating function (39) as 


$$
G(\boldsymbol{\gamma}, t)=\langle I \mid \rho(\gamma, t)\rangle=\sum_{m} \rho_{m}(\gamma, t),
$$

where $|I\rangle$ is the unit vector. Proceeding as in Sec. II, the formal solution of Eqs. (42) and (43) in Laplace space leads to the general solution for the generating function

$$
\tilde{G}(\gamma, s)=\left\langle I\left|\left[\hat{\widetilde{\Phi}}^{\prime}(s)+\hat{\tilde{\Phi}}(s)(\hat{I}-\hat{\tilde{\Psi}}(\boldsymbol{\gamma}, s))^{-1} \hat{\tilde{\Psi}}^{\prime}(\boldsymbol{\gamma}, s)\right]\right| \rho(0)\right\rangle .
$$

If Eq. (45) can be evaluated and inverse Laplace transformed, it provides the full statistics of the energy and matter transfer for a finite time interval. This is often a difficult task, and one therefore often focuses on the long-time behavior. This behavior is accessed through the solution (45) and also through the equation of motion for the generating function, which can be deduced by proceeding in the same way as in Sec. III when deriving the generalized master equation from Eqs. (42) and (43). Defining

$$
\widetilde{W}_{m m^{\prime}}^{(\nu)}(\boldsymbol{\gamma}, s) \equiv \frac{\widetilde{\psi}_{m m^{\prime}}^{(\nu)}(\boldsymbol{\gamma}, s)}{\widetilde{\phi}_{m^{\prime}}(s)},
$$

we find

$$
\begin{aligned}
\dot{\rho}_{m}(\boldsymbol{\gamma}, t)= & \mathcal{I}(\boldsymbol{\gamma}, t)+\sum_{m^{\prime}, \nu} \int_{0}^{t} d \tau\left[W_{m m^{\prime}}^{(\nu)}(\boldsymbol{\gamma}, \tau) \rho_{m^{\prime}}(\boldsymbol{\gamma}, t-\tau)\right. \\
& \left.-W_{m^{\prime} m}^{(\nu)}(\tau) \rho_{m}(\boldsymbol{\gamma}, t-\tau)\right],
\end{aligned}
$$

where

$$
\begin{aligned}
\tilde{\mathcal{I}}(\boldsymbol{\gamma}, s) \equiv & \sum_{m^{\prime}, \nu}\left\{\tilde{W}_{m m^{\prime}}^{(\nu)}(\boldsymbol{\gamma}, s)\left[\widetilde{\phi}_{m^{\prime}}(s)-\widetilde{\phi}_{m^{\prime}}(s)\right] \rho_{m^{\prime}}(0)\right. \\
& \left.-\widetilde{W}_{m^{\prime} m}^{(\nu)}(s)\left[\widetilde{\phi}_{m}(s)-\widetilde{\phi}_{m}^{\prime}(s)\right] \rho_{m}(0)\right\} .
\end{aligned}
$$

To calculate the long-time behavior of the moments of the probability distribution of heat and matter transfer between the system and its reservoir, we first consider the situation when a jump occurred at time zero. We will comment later on situations in which this is not the case. In the long-time limit, Eq. (45) diverges at $\gamma=0$ because $[\hat{I}-\hat{\widetilde{\Psi}}(s)]^{-1}=(\hat{I}$ $s \rightarrow 0$ $-\hat{P})^{-1}$, a limit which is singular because the determinant of $\hat{I}-\hat{P}$ is zero. This can be seen by considering the transpose of the matrix (transposition does not affect the determinant) and by replacing its first column by the sum of all the columns of the matrix (the determinant is not affected by replacing a column by a linear combination of it with other columns), which only contains zeros since $\Sigma_{m^{\prime} \neq m} P_{m^{\prime} m}=1$ (the determinant of a matrix with a zero column is zero). To lowest order in $s$, the determinant behaves like $\sim s^{\alpha}$. This means that at small $s,[\hat{I}-\hat{\tilde{\Psi}}(s)]^{-1} \sim s^{-\alpha}$. Using the long-time behavior of $s \rightarrow 0$

the WTD as expressed in Eqs. (1) and (26), and using Eq. (45), we thus see that $\widetilde{G}(\gamma=0, s) \sim s^{-1}$. We could have ar-

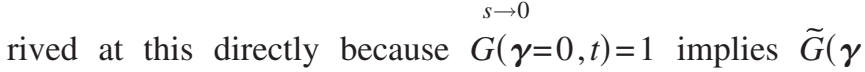

$=0, s)=s^{-1}$. This reasoning makes it clear that in order to calculate moments, the derivatives with respect to one of the $\gamma$ 's evaluated at $\gamma=0$ must be calculated from Eq. (45) before the long-time limit is taken.

Upon taking the $n$th derivative of Eq. (45) with respect to one of the $\gamma$ 's at $\gamma=0$, the dominant contribution at small $s$ is

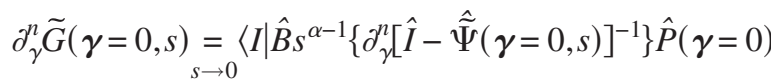

$$
\begin{aligned}
& \times|\rho(0)\rangle \sim s^{-n \alpha-1} .
\end{aligned}
$$

We used the fact that at small $s, \partial_{\gamma}^{n}[\hat{I}-\hat{\tilde{\Psi}}(\gamma=0, s)]^{-1}$ $\sim s^{-\alpha(n+1)} . \hat{B}$ is a diagonal matrix with diagonal elements $B_{m}$. Using Tauberian theorems $[32,45,46]$, we conclude that the moments of the energy and matter transfer between the system and the reservoir $\nu$ will behave at long times as

$$
\left\langle\left(\Delta E^{(\nu)}\right)^{n}\right\rangle,\left\langle\left(\Delta N^{(\nu)}\right)^{n}\right\rangle \underset{t \rightarrow \infty}{\sim} t^{n \alpha} .
$$

As noted earlier, these moments are experimentally accessible.

Let us now turn back to the case where no jump occurred at time zero. We only comment on WTDs with $\alpha=1$ since only then is it possible to carry out the averaging procedure described in Sec. II. Since $\widetilde{\Psi}_{m m^{\prime}}^{\prime}(s)=t_{m m^{\prime}} / t_{m^{\prime}}$ and $\widetilde{\Psi}_{m m^{\prime}}(s)=P_{m m^{\prime}}$, Eq. (50) with $\alpha=1$ is still valid, perhaps with a different proportionality factor. In the Appendix we explicitly implement these ideas by calculating the moments for a two level quantum dot.

\section{FLUCTUATION THEOREMS}

We now explore the conditions under which a fluctuation theorem holds for a CTRW. Two types of derivations have been used to obtain steady-state fluctuation theorems for stochastic dynamics. The first relies on a symmetry of the generating function, which translates into a fluctuation theorem using large deviation theory as in Refs. $[12,15]$. The other exploits the specific form of the logarithm of the ratio of the probability of a trajectory and the probability of its time reversed trajectory. We will use both for CTRWs and find that they can only be considered equivalent under specific conditions.

\section{A. Using large deviation}

This approach to arrive at a fluctuation theorem can only be implemented for $\alpha=1$ because it requires a finite mean waiting time between transitions. We thus restrict our discussion to this case. We have seen in Sec. III A that as long as one considers WTDs with $\alpha=1$, the generalized master equation at long times behaves like a Markovian master equation and thus reaches a steady state. Using the same arguments, we can show that the equation of motion (47) for the generating function behaves at long times as 


$$
\frac{\partial}{\partial t}|G(\boldsymbol{\gamma}, t)\rangle=\underset{t \rightarrow \infty}{W}(\boldsymbol{\gamma})|G(\boldsymbol{\gamma}, t)\rangle,
$$

where

$$
\begin{gathered}
{[\boldsymbol{W}(\boldsymbol{\gamma})]_{m m^{\prime}} \equiv \tilde{W}_{m m^{\prime}}(\boldsymbol{\gamma}, 0) \quad \text { for } m \neq m^{\prime},} \\
{[\boldsymbol{W}(\boldsymbol{\gamma})]_{m m} \equiv-\sum_{n} \tilde{W}_{n m}(0) .}
\end{gathered}
$$

This implies that for long times

$$
G(\gamma, t)=C(\gamma, t) e^{S(\gamma) t},
$$

where $\lim _{t \rightarrow \infty} \frac{1}{t} \ln C(\gamma, t)=0$ and where $S(\gamma)$ is the dominant eigenvalue of $\widetilde{W}(\boldsymbol{\gamma}, 0)$. This dominant eigenvalue gives the cumulant generating function because

$$
S(\gamma)=\lim _{t \rightarrow \infty} \frac{1}{t} \ln G(\gamma, t) .
$$

Note that the derivation to follow does not explicitly require the generalized master equation to be equivalent to a Markovian master equation at long times; what is required is the limiting behavior (54). Furthermore, using Eq. (33), we can verify that Eq. (52) satisfies

$$
\boldsymbol{W}(\boldsymbol{\gamma})=\boldsymbol{W}^{t}(\boldsymbol{A}-\boldsymbol{\gamma}),
$$

where $\boldsymbol{A}=\left(\left\{\beta_{\nu}\right\},\left\{-\beta_{\nu} \mu_{\nu}\right\}\right)$. Since these two matrices have the same eigenvalues, this implies the symmetry

$$
S(\boldsymbol{\gamma})=S(\boldsymbol{A}-\boldsymbol{\gamma}) .
$$

Large deviation theory $[12,15]$ can now be applied. The limiting behavior (54) and the symmetry (56) then imply the fluctuation theorem for the probability $P\left(\left\{-\frac{1}{t} \Delta E^{(\nu)}\right\}\right.$, $\left.\left\{-\frac{1}{t} \Delta N^{(\nu)}\right\}\right)$ for the energy and matter currents (cf. below) between the system and the reservoir $\nu$ at long times $[12,15]$,

$$
\frac{P\left(\left\{\frac{1}{t} \Delta E^{(\nu)}\right\},\left\{\frac{1}{t} \Delta N^{(\nu)}\right\}\right)}{P\left(\left\{-\frac{1}{t} \Delta E^{(\nu)}\right\},\left\{-\frac{1}{t} \Delta N^{(\nu)}\right\}\right)}=e^{\Delta S_{r}} \quad \text { for } t \rightarrow \infty .
$$

Here

$$
\Delta S_{r}=\sum_{\nu}\left(\beta_{\nu} \Delta E^{(\nu)}-\beta_{\nu} \mu_{\nu} \Delta N^{(\nu)}\right)
$$

represents the change of entropy due to the exchange processes with the reservoirs. We note that the change in energy and matter can be written in terms of energy and matter currents as

$$
\begin{aligned}
& \Delta E^{(\nu)} \equiv \int_{0}^{t} d \tau I_{\mathrm{e}}^{(\nu)}(\tau), \\
& \Delta N^{(\nu)} \equiv \int_{0}^{t} d \tau I_{\mathrm{m}}^{(\nu)}(\tau),
\end{aligned}
$$

so that in the long-time limit $\Delta E^{(\nu)} / t$ and $\frac{1}{t} \Delta N^{(\nu)} / t$ correspond to steady-state currents. This is why Eq. (57) is called a current fluctuation theorem [15].

We now define the matrix $\boldsymbol{W}(\bar{\gamma})$ as $\boldsymbol{W}(\boldsymbol{\gamma})$ where $\boldsymbol{\gamma}$ is replaced by $\bar{\gamma}=\left(\left\{\gamma_{\mathrm{e}} \beta_{\nu}\right\},\left\{-\gamma_{\mathrm{m}} \beta_{\nu} \mu_{\nu}\right\}\right)$. Obviously, when replac- ing $\boldsymbol{W}(\boldsymbol{\gamma})$ by $\boldsymbol{W}(\bar{\gamma})$ in our previous results, we calculate the statistics of $\Delta S_{r}$. The symmetry (55) now implies that $\boldsymbol{W}(\bar{\gamma})=\boldsymbol{W}^{t}(1-\bar{\gamma})$ so that

$$
S(\bar{\gamma})=S(1-\bar{\gamma}) .
$$

Using again large deviation theory, we get the fluctuation theorem

$$
\frac{P\left(\frac{1}{t} \Delta S_{r}\right)}{P\left(-\frac{1}{t} \Delta S_{r}\right)}=e^{\Delta S_{r}} \quad \text { for } t \rightarrow \infty .
$$

In the steady state, the average energy and matter transferred with a reservoir $\nu$ can be obtained by taking the derivative with respect to $\gamma_{\nu}$ at $\gamma=0$ of the formal solution of Eq. (51). We get

$$
\begin{gathered}
\left\langle\Delta E^{(\nu)}\right\rangle / t \equiv\left\langle I_{\mathrm{e}}^{(\nu)}\right\rangle=\sum_{m, m^{\prime}}\left(\epsilon_{m}-\epsilon_{m^{\prime}}\right) W_{m m^{\prime}}^{(\nu)} \rho_{m^{\prime}}^{\mathrm{ss}}, \\
\left\langle\Delta N^{(\nu)}\right\rangle / t \equiv\left\langle I_{\mathrm{m}}^{(\nu)}\right\rangle=\sum_{m, m^{\prime}}\left(N_{m}-N_{m^{\prime}}\right) W_{m m^{\prime}}^{(\nu)} \rho_{m^{\prime}}^{\mathrm{ss}} .
\end{gathered}
$$

Current conservation at steady state follows from

$$
\sum_{\nu}\left\langle I_{\mathrm{e}}^{(\nu)}\right\rangle=\sum_{\nu}\left\langle I_{\mathrm{m}}^{(\nu)}\right\rangle=0 .
$$

If we assume that all $M$ reservoirs $(\nu=1, \ldots, M)$ have different temperatures and chemical potentials, we have $M-1$ independent nonequilibrium forces associated with energy and matter transfer that can be defined as

$$
\begin{gathered}
X_{\mathrm{e}}^{(i)}=\beta_{1}-\beta_{i+1}, \\
X_{\mathrm{m}}^{(i)}=-\beta_{1} \mu_{1}+\beta_{i+1} \mu_{i+1},
\end{gathered}
$$

where $i=1, \ldots, M-1$. Using Eq. (64), the average change in the entropy due to exchange processes with the reservoirs $\left\langle\Delta S_{r}\right\rangle$ can be written in the familiar thermodynamical form of the entropy production in a steady state

$$
\left\langle\Delta S_{r}\right\rangle / t=\sum_{i=1}^{M-1}\left(X_{\mathrm{e}}^{(i)}\left\langle I_{\mathrm{m}}^{(i)}\right\rangle+X_{\mathrm{m}}^{(i)}\left\langle I_{\mathrm{m}}^{(i)}\right\rangle\right) .
$$

When $0<\alpha<1$, we have seen in Sec. III B that the solution of the generalized master equation behaves at long times as a power law $t^{-\alpha}$. This will also be the case for the solution of the equation of motion for the generating function (47). Therefore, contrary to Eq. (54), the cumulant generating function $\lim _{t \rightarrow \infty} \frac{1}{t} \ln G(\gamma, t)$ is zero. This indicates that the cumulants decay more slowly than $t$. The fluctuation theorem symmetry is only present in the eigenvalues of the generator of the generalized master equation (51) but not in the eigenvectors. For $\alpha=1$, the cumulant generating function is given by the dominant eigenvalue, so that the fluctuation theorem will reflect itself on all quantities that relate to it (large deviation theory is precisely used to make the link between this generating function and the probabilities). However, in the case $0<\alpha<1$, there is no way to separate in the long-time limit the contribution of the eigenvalues from the contribution of the eigenvectors to the statistics, thus preventing a fluctuation theorem from holding. 


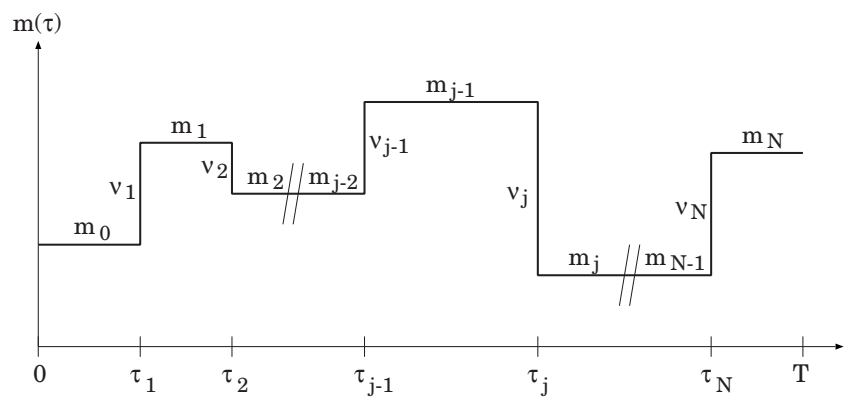

FIG. 1. Representation of a trajectory $m_{(\tau)}$.

\section{B. Using time reversal symmetry}

We denote a forward trajectory of the system between times $t=0$ and $t=T$ by $m_{\tau}$. As illustrated in Fig. 1, at $t=0$ the system is in state $m_{0}$ and stays there until it jumps to state $m_{1}$ at time $\tau_{1}$ via mechanism $\nu_{1}$. It remains there until time $\tau_{2}$, when it jumps to state $m_{2}$ via mechanism $\nu_{2}$. The trajectory continues in this fashion; at time $\tau_{N}$ there is a jump to state $m_{N}$, where the system remains at least until time $T$. The total number of jumps in this trajectory is $N$. The probability of this trajectory is

$$
P\left[m_{\tau}\right]=\rho_{m_{0}}(0) \psi_{m m^{\prime}}^{\prime\left(\nu_{1}\right)}\left(\tau_{1}\right)\left(\prod_{i=1}^{N-1} \psi_{m_{i+1} m_{i}}^{\left(\nu_{i+1}\right)}\left(\tau_{i+1}-\tau_{i}\right)\right) \phi_{m_{N}}\left(T-\tau_{N}\right) .
$$

The time-reversed trajectory $\bar{m}_{\tau}$ starts in state $m_{N}$ at time $T$, jumps to state $m_{N-1}$ at time $T-\tau_{N}$ via mechanism $\nu_{N}$, and so on. At time $T-\tau_{1}$ a jump to state $m_{1}$ occurs via mechanism $\nu_{1}$, and the system remains there until at least time 0 . The probability of this time reversed trajectory is

$P\left[\bar{m}_{\tau}\right]=\rho_{m_{N}}(T) \psi_{m_{N-1} m_{N}}^{\left(\nu_{N-1}\right)}\left(T-\tau_{N}\right)\left(\prod_{i=0}^{N-2} \psi_{m_{i} m_{i+1}}^{\left(\nu_{i}\right)}\left(\tau_{i+2}-\tau_{i+1}\right)\right) \phi_{m_{0}}\left(\tau_{1}\right)$.

Next we consider the quantity

$$
R\left[m_{\tau}\right] \equiv \ln \frac{P\left[m_{\tau}\right]}{P\left[\bar{m}_{\tau}\right]},
$$

whose explicit form reads

$$
\begin{aligned}
R\left[m_{\tau}\right]= & \ln \frac{\rho_{m_{0}}(0)}{\rho_{m_{N}}(T)}+\ln \prod_{i=1}^{N-1} \frac{\psi_{m_{i+1}}^{\left(\nu_{i+1}\right)}\left(m_{i}\right.}{\psi_{m_{i-1}}^{\left(\nu_{i}\right)}\left(\tau_{i}-\tau_{i+1}-\tau_{i}\right)} \\
& +\ln \frac{\psi_{m_{1} m_{0}}^{\prime\left(\nu_{1}\right)}\left(\tau_{1}\right) \phi_{m_{N}}\left(T-\tau_{N}\right)}{\psi_{m_{N-1}^{\prime} m_{N}}^{\prime\left(\nu_{N}\right)}\left(T-\tau_{N}\right) \phi_{m_{0}}\left(\tau_{1}\right)} .
\end{aligned}
$$

Because each $\bar{m}_{\tau}$ is the unique mirror image of $m_{\tau}$, a sum over all possible forward trajectories is equivalent to a sum over all possible time-reversed trajectories. Therefore since normalization implies that $\Sigma_{\bar{m}_{\tau}} P\left[\bar{m}_{\tau}\right]=1$, an integral fluctuation theorem follows immediately from the definition (69),

$$
\left\langle e^{-R}\right\rangle=\sum_{m_{\tau}} e^{-R\left[m_{\tau}\right]} P\left[m_{\tau}\right]=1 .
$$

The positivity of the ensemble average of $R\left[m_{\tau}\right],\langle R\rangle \geq 0$, then follows from Jensen's inequality. Using the important property $R\left[m_{\tau}\right]=-R\left[\bar{m}_{\tau}\right]$, which follows from Eq. (69) together with the fact that by taking twice the time reversal of a trajectory we get back to the original trajectory $\overline{\bar{m}}_{\tau}=m_{\tau}$, we can also derive a detailed fluctuation theorem for $R$,

$$
\begin{aligned}
P(R) & =\sum_{m_{\tau}} \delta\left(R-R\left[m_{\tau}\right]\right) P\left[m_{\tau}\right] \\
& =\sum_{m_{\tau}} \delta\left(R-R\left[m_{\tau}\right]\right) e^{R\left[m_{\tau}\right]} P\left[\bar{m}_{\tau}\right] \\
& =e^{R} \sum_{\bar{m}_{\tau}} \delta\left(R-R\left[m_{\tau}\right]\right) P\left[\bar{m}_{\tau}\right] \\
& =e^{R} \sum_{\bar{m}_{\tau}} \delta\left(R+R\left[\bar{m}_{\tau}\right]\right) P\left[\bar{m}_{\tau}\right]=e^{R} P(-R) .
\end{aligned}
$$

The integral fluctuation theorem (71) and the detailed fluctuation theorem (72) for $R$ are thus completely general and valid for any continuous-time random walk. It is worth mentioning that a similar derivation can be done for any dynamics as long as each trajectory has a corresponding timereversed trajectory with a nonzero probability [10].

To make these fluctuation theorems useful, we need to give a physical interpretation to $R\left[m_{\tau}\right]$. In particular, we will argue that $R$ can only be interpreted as a change of entropy if two conditions are satisfied. One is that the WTDs have a finite first moment $(\alpha=1)$. The other is that they be separable $[47,48]$, that is, that the waiting time distributions can be written as the product of a waiting time portion that depends only on the originating state, and a transition matrix that connects given initial and final states,

$$
\psi_{m m^{\prime}}^{(\nu)}(t)=P_{m m^{\prime}}^{(\nu)} \psi_{m^{\prime}}(t) .
$$

The separability condition is called "direction time independence" by Qian and Wang [47,48]. The first term of Eq. (70),

$$
\Delta S\left[m_{\tau}\right] \equiv \ln \frac{\rho_{m_{0}}(0)}{\rho_{m_{N}}(T)}=S_{m_{N}}(T)-S_{m_{0}}(0),
$$

where $S_{m}(t)=-\ln \rho_{m}(t)$, can be interpreted as a change of system (Gibbs) entropy along the trajectory because it depends only on the initial and final microscopic states of the system for that trajectory, and the average over trajectories is then simply $\langle\Delta S\rangle=S(t)-S(0)$, where $S(t)=\Sigma_{m} \rho_{m}(t) S_{m}(t)$ is just a straightforward average over states.

In order to interpret the two remaining terms in Eq. (70), we implement separability of the WTDs so that

$$
\begin{aligned}
R\left[m_{\tau}\right]= & \ln \frac{\rho_{m_{0}}(0)}{\rho_{m_{N}}(T)}+\ln \left(\prod_{i=0}^{N-1} \frac{P_{m_{i+1} m_{i}}^{\left(\nu_{i+1}\right)}}{P_{m_{i+1} m_{i+1}}^{\left(\nu_{i+1}\right)}}\right) \\
& +\ln \frac{\psi_{m_{0}}^{\prime}\left(\tau_{1}\right) \phi_{m_{N}}\left(T-\tau_{N}\right)}{\psi_{m_{N}}^{\prime}\left(T-\tau_{N}\right) \phi_{m_{0}}\left(\tau_{1}\right)} .
\end{aligned}
$$

Now only the WTDs of the first jumps remain. In Eq. (72), 
the path summation runs over all possible trajectories including those with or without a jump at time zero. One way to handle the problem of having to treat the first jump differently from the others is via the time averaging procedure (10) in Eq. (67) as well as in Eq. (68). This can only be done if the first moments are finite. The third term in Eq. (75) now becomes equal to $\ln \left(t_{m_{N}} / t_{m_{0}}\right)$, so that using Eq. (27) the second and third terms in Eq. (75) can be combined and $R\left[m_{\tau}\right]$ can be written in terms of the transition matrix elements as

$$
R\left[m_{\tau}\right]=\ln \frac{\rho_{m_{0}}(0)}{\rho_{m_{N}}(T)}+\ln \left(\prod_{i=0}^{N-1} \frac{\widetilde{W}_{m_{i+1}}^{\left(\nu_{i+1}\right)}(0)}{\widetilde{W}_{m_{i} m_{i+1}}^{\left(\nu_{i+1}\right)}(0)}\right) .
$$

This form of $R\left[m_{\tau}\right]$ is now exactly the same as the one derived for the Markovian master equation (29) (see [14,23]). This is a manifestation of the so-called corresponding Markov process of a CTRW [48].

We denote the second term on the right-hand side of Eq. (76) as $\Delta S_{r}\left[m_{\tau}\right]$ and call it the reservoir part of the trajectory entropy because it can be interpreted as the change in entropy along the trajectory due to exchange processes between the system and the reservoirs. Indeed, using Eq. (33), this term can be expressed as

$$
\begin{aligned}
\Delta S_{r}\left[m_{\tau}\right] & \equiv \ln \left(\prod_{i=0}^{N-1} \frac{\tilde{W}_{m_{i+1} m_{i}}^{\left(\nu_{i+1}\right)}(0)}{\tilde{W}_{m_{i} m_{i+1}}^{\left(\nu_{i+1}\right)}(0)}\right) \\
& =\sum_{\nu}\left(-\beta_{\nu} \Delta E^{(\nu)}\left[m_{\tau}\right]+\beta_{\nu} \mu_{\nu} \Delta N^{(\nu)}\left[m_{\tau}\right]\right),
\end{aligned}
$$

where the change of energy and number of particles along the trajectory due to the mechanism $\nu$ can be expressed in terms of heat and matter currents along the trajectory as

$$
\begin{aligned}
\Delta E^{(\nu)}\left[m_{\tau}\right] & \equiv \int_{0}^{t} d \tau I_{e}^{(\nu)}\left[m_{\tau}\right], \\
\Delta N^{(\nu)}\left[m_{\tau}\right] & \equiv \int_{0}^{t} d \tau I_{m}^{(\nu)}\left[m_{\tau}\right]
\end{aligned}
$$

[cf. Eqs. (59) and (60)]. These currents are sequences of $\delta$ functions centered at the times of the jumps and multiplied by the corresponding energy or matter change. They are positive (negative) if energy or matter increases (decreses) in the system.

We have thus arrived at the important result that for separable WTDs with $\alpha=1, R\left[m_{\tau}\right]=\Delta S\left[m_{\tau}\right]+\Delta S_{r}\left[m_{\tau}\right]$. Since $\Delta S\left[m_{\tau}\right]$ and $\Delta S_{r}\left[m_{\tau}\right]$ are interpreted, respectively, as the change in the system entropy along the trajectory and the change in entropy due to the exchange processes between the system and its reservoirs, it is natural to interpret $R\left[m_{\tau}\right]$ as the total change in entropy along the trajectory (also called the total change in the trajectory entropy production) $[14,23]$. Note that in this case the principle of microreversibility, implying that at equilibrium the probability of a forward trajectory is identical to the probability of its time reversed trajectory $\left(R\left[m_{\tau}\right]=0\right)$, is satisfied if the detailed balance condition (32) is satisfied and if the system is initially at equilibrium [so that $\rho_{m_{0}}(0)=\rho_{m_{0}}^{\mathrm{eq}}$ and $\rho_{m_{N}}(T)=\rho_{m_{T}}^{\mathrm{eq}}$. This result is consis- tent with the findings of Ref. [48] stating that separability of the WTDs and detailed balance are sufficient conditions for microreversibility to be satisfied in a CTRW.

In Sec. V A we showed that a fluctuation theorem for $\Delta S_{r}$ can be derived for long times [see Eq. (62)]. In fact, considering separable WTDs with $\alpha=1$, the fluctuation theorem (62) can be seen as resulting from the fluctuation theorem (72), as follows. The quantity $\Delta S\left[m_{\tau}\right]$ is a bounded quantity which only depends on the probability distribution of the initial and final states of the trajectory. On the contrary, $\Delta S_{r}\left[m_{\tau}\right]$ changes each time a jump occurs along the system trajectory. It is therefore reasonable to assume that for very long trajectories and for the huge majority of realizations, the contribution from $\Delta S_{r}\left[m_{\tau}\right]$ in $R\left[m_{\tau}\right]=\Delta S\left[m_{\tau}\right]+\Delta S_{r}\left[m_{\tau}\right]$ will be significantly dominant so that in the long-time limit Eq. (72) reduces to Eq. (62). However, the derivation of Eq. (62) only required WTDs with $\alpha=1$, but did not require separability. The reason for this requirement in order to identify Eq. (72) as a useful fluctuation theorem for all times is that otherwise $R\left[m_{\tau}\right]$ has no clear physical interpretation. The problem is that $R\left[m_{\tau}\right]$ [and, more specifically, the second term in Eq. (70), which according to our previous argument dominates at long times] depends on the time intervals between the jumps along the trajectory. This implies that at the level of a single trajectory, $R\left[m_{\tau}\right]$ cannot be expressed in terms of exchange processes with the reservoirs (more precisely, in terms of time integrated currents). However, the fluctuation theorem (62) indicates that at long times the probability $P(R)$ to observe a trajectory such that $R\left[m_{\tau}\right]=R$ becomes equivalent to the probability to observe a trajectory with a set of time integrated currents so that, via Eq. (58), $\Delta S_{r} \approx R$. One way to understand this result is to coarse-grain the trajectories in time. Instead of specifying exactly the time at which each jump occurs, we define small time intervals of equal size $d t$ (sufficiently small so that the probability of observing two transitions in one is interval negligible, i.e., small compared to the mean time for a transition to occur). We then specify whether a transition between two states occurred or not in this interval. In this way, we define coarse-grained trajectories (denoted by $\widetilde{m}_{\tau}$ ), and we note that different microscopic trajectories can lead to the same coarse-grained trajectory. To calculate the probabilities of these trajectories, we use the fact that at long times the dynamics is described by Eq. (29), which can be discretized in time intervals $d t$. This discretized form allows us to identify the probability to stay in a given state $m$ during a given time interval with 1 $-\Sigma_{m^{\prime}, \nu} \widetilde{W}_{m^{\prime} m}^{\nu}(0) d t$, and the probability to jump from $m$ to $m^{\prime}$ by mechanism $\nu$ with $\widetilde{W}_{m m^{\prime}}^{\nu}(0) d t$. Using these probabilities, we can construct the probability of a coarse-grained trajectory and that of its time reversed coarse-grained trajectory. The logarithm of their ratio gives $\Delta S\left[\tilde{m}_{\tau}\right]+\Delta S_{r}\left[\tilde{m}_{\tau}\right]$, with the same definitions as in Eqs. (74) and (77), but for $\widetilde{m}_{\tau}$ instead of $m_{\tau}$. With the argument that at long times $\Delta S_{r}\left[\tilde{m}_{\tau}\right]$ will be dominant, we understand why the fluctuation theorem (62) holds at long times. One should note that long times are also needed in this case to get rid of the contribution from the initial part of the trajectory where Eq. (29) is not valid. Also, contrary to the case where separability holds, it is only at the coarse-grained level and considering very long trajectories 
that such a dynamics can satisfy "microscopic" reversibility. The fluctuation theorem (62) does not result from a probabilistic asymmetry under time reversal at the microscopic trajectory level as in the case of separable WTDs, but at a coarse-grained level and only for long times.

We can finally comment on the case of WTDs with diverging mean waiting times. Even if separability is satisfied, because mean waiting times diverge, Eq. (33) cannot be used to express $R\left[m_{\tau}\right]$ (or parts of it) in terms of exchange processes with the reservoirs. The fluctuation theorem (72) is thus valid but seemingly useless.

\section{CONCLUSIONS}

In this paper we have considered continuous time random walks (CTRWs) in which multiple mechanisms that may have different statistical properties induce transitions between pairs of states. A given energy and number of particles can be attributed to each state, so that each mechanism can be thought of as corresponding to a given reservoir. If these reservoirs have different statistical properties, such a CTRW therefore describes the stochastic dynamics of an open system. The statistics of the transitions between states associated with each mechanism are described by waiting time distributions (WTDs). If a WTD decays at long times at least as fast as $t^{-2}$, the distribution has a finite first moment and we say that $\alpha=1$. If the decay is slower, as $t^{-\alpha-1}$ where $0<\alpha$ $<1$, all moments diverge.

We have analyzed the long-time behavior of the probability $\rho_{m}(t)$ that the system is in state $m$ at time $t$ via the generalized master equation for this probability. When $\alpha=1$ the generalized master equation leads at long times to an exponential decay of the probability to a steady state, exactly as it would for an ordinary Markovian master equation. If the WTDs corresponding to the different mechanisms are different, the steady state is a nonequilibrium steady state. If they are identical, the steady state distribution corresponds to equilibrium and satisfies detailed balance. On the other hand, if $0<\alpha<1$, the probability $\rho_{m}(t)$ evolves as a linear combination of power-law decays $t^{-\alpha}$ toward the zero eigenvalue mode, and the system never reaches a steady state.

We have presented a formalism to calculate the counting statistics of the energy and matter transfer in the CTRW based on a generating function propagation method. By considering systems exchanging energy and matter with different reservoirs, we have shown that the $n$th moment of the probability distribution to exchange a certain amount of energy or matter between the system and a given reservoir during a time interval $t$ behaves as $t^{n \alpha}$ at long times. This result holds for $0<\alpha \leq 1$ and reflects the subordination principle $[49,50]$.

Using our generating function formalism together with large deviation theory, for WTDs with $\alpha=1$ we derived a fluctuation theorem for the trajectory quantity $\Delta S_{r}\left[m_{\tau}\right]$ representing the change of entropy along the trajectory due to exchange processes with the reservoirs [which can be explicitly related to the energy and matter currents between the system and the reservoirs (58)]. If $P\left(\Delta S_{r}\right)$ is the probability to observe a trajectory along which occurs a change
$\Delta S_{r}\left[m_{\tau}\right]=\Delta S_{r}$, the fluctuation theorem reads $P\left(\Delta S_{r}\right) /$ $P\left(-\Delta S_{r}\right)=\exp \left\{\Delta S_{r}\right\}$ and is only valid at long times. For WTDs with diverging first moments, $0<\alpha<1$, this fluctuation theorem does not hold.

The trajectory quantity $R\left[m_{\tau}\right]$ is defined as the logarithm of the ratio of the probability of a given trajectory to the time reversed trajectory. We have shown that for any CTRW, the ensemble average of $R\left[m_{\tau}\right]$ is always positive and a fluctuation theorem stating that the probability $P(R)$ to observe a trajectory such that $R\left[m_{\tau}\right]=R$ is exponentially more likely than to observe a trajectory such that $R\left[m_{\tau}\right]=-R$, i.e., $P(R) / P(-R)=\exp \{R\}$ always holds. Separable WTDs are ones for which the state-directional part and the temporal part of the WTD factorize [see Eq. (73)]. We have shown that it is only for separable WTDs with $\alpha=1$ that $R\left[m_{\tau}\right]$ can be interpreted as the change of entropy along the trajectory. In this case, this fluctuation theorem is related to the previous one because $R\left[m_{\tau}\right]=\Delta S\left[m_{\tau}\right]+\Delta S_{r}\left[m_{\tau}\right]$, where $\Delta S\left[m_{\tau}\right]$ is the change of system entropy along the trajectory and is bounded, contrary to $\Delta S_{r}\left[m_{\tau}\right]$ which typically grows for long trajectories. Therefore for most realizations $R\left[m_{\tau}\right]$ $\approx \Delta S_{r}\left[m_{\tau}\right]$ at long times, thus providing an interpretation of the fluctuation theorem for $\Delta S_{r}\left[m_{\tau}\right]$ in terms of probabilistic asymmetry under time reversal of the probability of a trajectory. At equilibrium, the symmetry is restored and $R\left[m_{\tau}\right]=0$ as expected from the principle of microreversibility. For nonseparable WTDs with $\alpha=1, R\left[m_{\tau}\right]$ can only be related to a change of entropy after a coarse graining of the trajectories in time. The fluctuation theorem for $\Delta S_{r}\left[m_{\tau}\right]$ still holds, but can only be interpreted as a measure of a probabilistic asymmetry in time at the level of coarse-grained trajectory (and also only for long times), and not at the level of the microscopic trajectories as in the case of separable WTDs.

\section{ACKNOWLEDGMENTS}

M. E. would like to thank František Šanda and Shaul Mukamel for early discussions which motivated this work. M. E. was supported by the FNRS Belgium (chargé de recherches) and by the Luxemburgish government (Bourse de formation recherches). This research was supported in part by the National Science Foundation under Grant No. PHY0354937.

\section{APPENDIX: SINGLE LEVEL QUANTUM DOT}

As an application of the counting statistics results of Sec. IV, we consider a single level quantum dot between left and right leads $\nu=L, R$. There are two states in the system, one corresponding to the empty level $m=0$ and the other to the filled level $m=1$. Since the energy transfer between the system and a reservoir is directly proportional to the particle transfer in this model, we only consider particle transfer. We take separable WTDs, specifically

$$
\begin{aligned}
& \psi_{00}(\boldsymbol{\gamma}, t)=\psi_{11}(\boldsymbol{\gamma}, t)=0, \\
& \psi_{10}(\boldsymbol{\gamma}, t)=P_{10}(\boldsymbol{\gamma}) \psi_{0}(t), \\
& \psi_{01}(\boldsymbol{\gamma}, t)=P_{01}(\boldsymbol{\gamma}) \psi_{1}(t),
\end{aligned}
$$


where

$$
\begin{gathered}
P_{10}(\gamma)=e^{\gamma^{(L)}} P_{10}^{(L)}+e^{\gamma^{(R)}} P_{10}^{(R)}, \\
P_{01}(\gamma)=e^{-\gamma^{(L)}} P_{01}^{(L)}+e^{-\gamma^{(R)}} P_{01}^{(R)} .
\end{gathered}
$$

Note that

$$
\begin{aligned}
& P_{10}(\gamma=0)=P_{10}^{(L)}+P_{10}^{(R)}=1, \\
& P_{01}(\gamma=0)=P_{01}^{(L)}+P_{01}^{(R)}=1 .
\end{aligned}
$$

We define the affinities $A_{x} \equiv \ln P_{01}^{(x)} / P_{10}^{(x)}$, where $x=L, R$, and $\Delta A \equiv A_{L}-A_{R}$.

From now on, we focus on the net particle transfer between the left lead and the dot so that $\gamma^{(L)}=\gamma$ and $\gamma^{(R)}=0$. We also note that

$$
\begin{gathered}
P_{01}(\gamma) \equiv e^{A_{R}} P_{10}(\Delta A-\gamma), \\
P_{10}(\gamma) \equiv e^{-A_{R}} P_{01}(\Delta A-\gamma) .
\end{gathered}
$$

The generating function (45) for this model reads

$$
\begin{aligned}
\widetilde{G}(\gamma, s)= & \left(\widetilde{\phi}_{0}^{\prime}(s)\right. \\
& \left.+\widetilde{\psi}_{0}^{\prime}(s) \frac{\widetilde{\phi}_{1}(s) P_{10}(\gamma)+\widetilde{\psi}_{1}(s) \widetilde{\phi}_{0}(s) P_{01}(\gamma) P_{10}(\gamma)}{1-P_{01}(\gamma) P_{10}(\gamma) \tilde{\psi}_{0}(s) \widetilde{\psi}_{1}(s)}\right) \\
& \times \rho_{0}(0)+(1 \leftrightarrow 0) .
\end{aligned}
$$

We can verify that the generating function at $\gamma=0$ diverge as $s^{-1}$ in the long-time limit, and, because of Eq. (A4), the same is true at $\gamma=\Delta A$. The first moment reads

$$
\left.\partial_{\gamma} \tilde{G}(\gamma, s)\right|_{\gamma=0}=\frac{\widetilde{\phi}_{0}(s) \tilde{\psi}_{1}(s)\left(P_{10}^{(L)}-P_{01}^{(L)}\right)+\widetilde{\phi}_{1}(s)\left[P_{10}^{(L)}-\widetilde{\psi}_{0}(s) \tilde{\psi}_{1}(s) P_{01}^{(L)}\right]}{\left[\tilde{\psi}_{0}(s) \tilde{\psi}_{1}(s)-1\right]^{2}} \widetilde{\psi}_{0}^{\prime}(s) \rho_{0}(0)-(1 \leftrightarrow 0) .
$$

The WTDs are taken to be of the form

$$
\begin{aligned}
& \widetilde{\psi}_{0}(s)=1-B_{0} s^{\alpha}, \\
& \tilde{\psi}_{1}(s)=1-B_{1} s^{\alpha},
\end{aligned}
$$

where $0 \leq \alpha \leq 1$. If $\alpha=1, B_{0}=t_{0}$ and $B_{1}=t_{1}$.

We can now confirm that if $0<\alpha<1$ and a jump occurred at time zero $\left[\psi_{m}^{\prime}(t)=\psi_{m}(t)\right]$, or if $\alpha=1$ and a jump occurred at time zero $\left[\psi_{m}^{\prime}(t)=\psi_{m}(t)\right]$, or if $\alpha=1$ and we do not know at time zero when the last jump occurred $\left[\widetilde{\psi}_{m}^{\prime}(s)=\widetilde{\phi}_{m}(s) / t_{m}\right]$, we always get

$$
\left.\partial_{\gamma}^{n} \widetilde{G}(\gamma, s)\right|_{\gamma=0}=\frac{n !\left(P_{10}^{(L)}-P_{01}^{(L)}\right)^{n}}{\left(B_{0}+B_{1}\right)^{n}} s^{-n \alpha-1},
$$

where $n=1,2,3, \ldots$ Using the Tauberian theorem, this leads to

[1] J. Liphardt, S. Dumont, S. B. Smith, I. Tinoco, Jr., and C. Bustamante, Science 296, 1832 (2002).

[2] E. H. Trepagnier, C. Jarzynski, F. Ritort, G. E. Crooks, C. J. Bustamante, and J. Liphardt, Proc. Natl. Acad. Sci. U.S.A. 101, 15038 (2004).

[3] D. Collin, F. Ritort, C. Jarzynski, S. B. Smith, I. Tinoco, Jr., and C. Bustamante, Nature (London) 437, 231 (2005).

[4] G. M. Wang, J. C. Reid, D. M. Carberry, D. R. M. Williams, E. M. Sevick, and D. J. Evans, Phys. Rev. E 71, 046142 (2005).

$$
\left.\partial_{\gamma}^{n} G(\gamma, t)\right|_{\gamma=0}=\frac{n !\left(P_{10}^{(L)}-P_{01}^{(L)}\right)^{n}}{\Gamma(n \alpha+1)\left(B_{0}+B_{1}\right)^{n}} t^{n \alpha} .
$$

When $\alpha=1$, Eq. (A9) can be written as

$$
\left.\partial_{\gamma}^{n} G(\gamma, t)\right|_{\gamma=0}=\left(\frac{W_{10}^{L} W_{01}-W_{01}^{L} W_{10}}{W_{10}+W_{01}}\right)^{n} t^{n \alpha},
$$

where $W_{m m^{\prime}}^{(\nu)}=P_{m m^{\prime}}^{(\nu)} / t_{m^{\prime}}$ and $W_{m m^{\prime}}=W_{m m^{\prime}}^{(L)}+W_{m m^{\prime}}^{(R)}$. This is the same result as obtained in Ref. [23] using a Markovian master equation. In can easily be seen that Eq. (A10) vanishes when detailed balance is satisfied.

Finally, we note that the cumulants calculated from these moments all vanish. This indicates that we should calculate not only the leading time dependence of the moments as we do when applying Tauberian theorems, but must retain higher orders to extract information about the time dependence of the cumulants.
[5] S. Schuler, T. Speck, C. Tietz, J. Wrachtrup, and U. Seifert, Phys. Rev. Lett. 94, 180602 (2005).

[6] C. Tietz, S. Schuler, T. Speck, U. Seifert, and J. Wrachtrup, Phys. Rev. Lett. 97, 050602 (2006).

[7] D. Andrieux, P. Gaspard, S. Ciliberto, N. Garnier, S. Joubaud, and A. Petrosyan, Phys. Rev. Lett. 98, 150601 (2007).

[8] C. Jarzynski, Phys. Rev. Lett. 78, 2690 (1997); Phys. Rev. E 56, 5018 (1997).

[9] R. Kawai, J. M. R. Parrondo, and C. Van den Broeck, Phys. 
Rev. Lett. 98, 080602 (2007).

[10] B. Cleuren, C. Van den Broeck, and R. Kawai, C. R. Phys. 8, 567 (2007).

[11] J. Kurchan, J. Phys. A 31, 3719 (1998).

[12] J. L. Lebowitz and H. Spohn, J. Stat. Phys. 95, 333 (1999).

[13] G. E. Crooks, Phys. Rev. E 60, 2721 (1999); 61, 2361 (2000).

[14] U. Seifert, Phys. Rev. Lett. 95, 040602 (2005).

[15] D. Andrieux and P. Gaspard, J. Stat. Phys. 127, 107 (2007).

[16] M. Esposito, U. Harbola, and S. Mukamel, Phys. Rev. E 76, 031132 (2007).

[17] D. J. Evans and D. J. Searles, Phys. Rev. E 50, 1645 (1994); 52, 5839 (1995); 53, 5808 (1996).

[18] G. Gallavotti and E. G. D. Cohen, Phys. Rev. Lett. 74, 2694 (1995); J. Stat. Phys. 80, 931 (1995).

[19] J. Kurchan, e-print arXiv:cond-mat/0007360.

[20] H. Tasaki, e-print arXiv:cond-mat/0009244.

[21] S. Mukamel, Phys. Rev. Lett. 90, 170604 (2003).

[22] P. Talkner and P. Hanggi, J. Phys. A 40, F569 (2007).

[23] M. Esposito, U. Harbola, and S. Mukamel, Phys. Rev. B 75, 155316 (2007).

[24] B. Cleuren and C. Van den Broeck, Europhys. Lett. 79, 30001 (2007).

[25] F. Zamponi, F. Bonetto, L. F. Cugliandolo, and J. Kurchan, J. Stat. Mech.: Theory Exp. (2005), P09013.

[26] T. Ohkuma and T. Ohta, J. Stat. Mech.: Theor. Exp. (2007), P10010.

[27] E. W. Montroll and G. H. Weiss, J. Math. Phys. 6, 167 (1965).

[28] H. Scher and M. Lax, Phys. Rev. B 7, 4491 (1973); 7, 4502 (1973).

[29] J. W. Haus and K. W. Kehr, Phys. Rep. 150, 263 (1987).

[30] J.-P. Bouchaud and A. Georges, Phys. Rep. 195, 127 (1990).

[31] D. Bedeaux, K. Lakatos-Lindenberg, and K. Shuler, J. Math. Phys. 12, 2116 (1971).

[32] B. D. Hughes, Random Walks and Random Environments: Volume 1: Random Walks (Oxford University Press, New York, 1995); B. D. Hughes, Random Walks and Random Environ- ments: Volume 2: Random Environments (Oxford University Press, New York, 1996).

[33] J. Schnakenberg, Rev. Mod. Phys. 48, 571 (1976).

[34] E. Barkai and Y. C. Cheng, J. Chem. Phys. 118, 6167 (2003).

[35] P. Allegrini, G. Aquino, P. Grigolini, L. Palatella, and A. Rosa, Phys. Rev. E 68, 056123 (2003).

[36] W. Ebeling and I. M. Sokolov, Statistical Thermodynamics and Stochastic Theory of Nonequilibrium Systems (World Scientific, Singapore, 2005).

[37] G. Bel and E. Barkai, Phys. Rev. E 73, 016125 (2006).

[38] A. Rebenshtok and E. Barkai, Phys. Rev. Lett. 99, 210601 (2007).

[39] Y. Jung, E. Barkai, and R. J. Silbey, Chem. Phys. 284, 181 (2002).

[40] X. Brokmann, J.-P. Hermier, G. Messin, P. Desbiolles, J.-P. Bouchaud, and M. Dahan, Phys. Rev. Lett. 90, 120601 (2003).

[41] H. Yang, G. Luo, P. Karnchanaphanurach, T. M. Louie, I. Rech, S. Cova, L. Xun, and X. S. Xie, Science 302, 262 (2003).

[42] O. Flomenbom, K. Velonia, D. Loos, S. Masuo, M. Cotlet, Y. Engelborghs, J. Hofkens, A. E. Rowan, R. J. M. Nolte, M. Van der Auweraer, F. C. De Schryver, and J. Klafter, Proc. Natl. Acad. Sci. U.S.A. 102, 2368 (2005).

[43] M. F. Shlesinger, J. Stat. Phys. 10, 421 (1974).

[44] R. Metzler and J. Klafter, Phys. Rep. 339, 1 (2000).

[45] W. Feller, An Introduction to Probability Theory and its Applications, 2nd ed. (Wiley, New York, 1971), Vol. II.

[46] J. Korevaar, Tauberian Theory: A Century of Developments (Springer, Berlin, 2004).

[47] H. Wang and H. Qian, J. Math. Phys. 48, 013303 (2007).

[48] H. Qian and H. Wang, Europhys. Lett. 76, 15 (2006).

[49] I. M. Sokolov and J. Klafter, Chaos 15, 026103 (2005).

[50] M. M. Meerschaert, D. A. Benson, H.-P. Scheffler, and B. Baeumer, Phys. Rev. E 65, 041103 (2002); A. A. Satinislavsky, Physica A 318, 469 (2003); R. Gorenflo, F. Mainardi, and A. Vivoli, Chaos, Solitons Fractals 34, 89 (2007). 